\title{
BTEB2 knockdown suppresses neointimal hyperplasia in a rat artery balloon injury model
}

\author{
DE LI* ${ }^{*}$, SHUANGTAO MA*, YONGJIAN YANG, DACHUN YANG, \\ GANG LI, XIN ZHANG, JUN ZHU, MEIQIN SUN and BING TANG \\ Department of Cardiology, General Hospital of PLA Chengdu Military Area Command, Sichuan 610083, P.R. China
}

Received October 26, 2010; Accepted February 8, 2011

DOI: $10.3892 / \mathrm{mmr} .2011 .438$

\begin{abstract}
Basic transcription element-binding protein 2 (BTEB2) is a regulator of the proliferation and phenotypic changes of vascular smooth muscle cells (SMCs). The aim of the present study was to determine whether or not BTEB2 knockdown inhibits balloon injury-induced neointimal hyperplasia attributed to the proliferation and phenotypic changes of vascular SMCs. We found that the knockdown of BTEB2 with antisense oligonucleotides (Ad-As-BTEB2) significantly reduced the intima/media ratio compared to uninjured arteries and vessels treated with Ad-LacZ. Knockdown of BTEB2 suppresses the proliferation of cultured vascular SMCs, concurrent with the down-regulation of proliferating cell nuclear antigen, angiotensin II type 1 receptor and plateletderived growth factor BB. In addition, BTEB2 knockdown caused the up-regulation of the differentiation marker smooth muscle $\alpha$-actin and down-regulation of the dedifferentiation marker embryonic smooth muscle myosin heavy chain. The present study provides direct evidence that BTEB2 plays a critical role in balloon injury-induced neointimal hyperplasia, which is closely linked to vascular SMC proliferation and phenotypic modulation. This study highlights the fact that BTEB2 may be a potential target for the prevention of restenosis after vascular intervention.
\end{abstract}

\section{Introduction}

Percutaneous coronary intervention (PCI) is widely used for the treatment of coronary atherosclerotic disease (1). However, the fact that balloon injury to the arterial wall induces restenosis presents clinical challenges $(2,3)$. The restenotic

Correspondence to: Dr Bing Tang, Department of Cardiology, General Hospital of PLA Chengdu Military Area Command, 270 Rongdu Rd., Tianhui, Jinniu, Chengdu, Sichuan 610083, P.R. China E-mail: dr_tangb@yahoo.cn

\section{${ }^{*}$ Contributed equally}

Key words: basic transcription factor binding protein 2, neointimal hyperplasia process is characterized by neointimal hyperplasia that results from the proliferation and migration of medial vascular smooth muscle cells (SMCs) (4,5). Therefore, the ability to modulate the proliferation and phenotypic changes of vascular SMCs may be a viable and novel strategy for the prevention of restenosis.

Basic transcription element-binding protein 2 (BTEB2), a transcription factor that binds GC boxes, is preferentially expressed in dedifferentiated or activated SMCs (6). It has been reported that atherosclerotic lesions containing BTEB2positive cells are associated with a higher risk of restenosis than BTEB2-negative lesions (7), suggesting that BTEB2 serves as a regulator of vascular SMC proliferation and phenotypic changes. It has also been demonstrated that BTEB2 expression increases over time during the course of vascular stenosis $(8,9)$, indicating that the induced expression of BTEB2 may play an important role in the development of restenosis after PCI by stimulating proliferation and phenotypic changes in vascular SMCs.

Based on these findings, we hypothesized that knockdown of BTEB2 suppresses vascular restenosis. In this study, we examined whether BTEB2 knockdown is capable of suppressing neointimal hyperplasia in a rat balloon injury model. We also evaluated the effect of BTEB2 knockdown on cultured vascular SMC proliferation and the expression of proliferation-related molecules, including proliferating cell nuclear antigen (PCNA), angiotensin II type 1 receptor (AT1R) and platelet-derived growth factor BB (PDGF-BB). In addition, we evaluated vascular SMC phenotypic modulations through the expression of the differentiation marker smooth muscle $\alpha$-actin (SM $\alpha \mathrm{A})$ and the dedifferentiation marker embryonic smooth muscle myosin heavy chain (SMemb). The results suggested that the inhibition of BTEB2 may provide a novel strategy for the treatment of vascular diseases.

\section{Materials and methods}

Construction of the Ad-As-BTEB2 vector. A 662-bp cDNA fragment of BTEB2 was generated by reverse transcriptionpolymerase chain reaction (RT-PCR) with specific primers (Invitrogen, Carlsbad, CA, USA). The forward primer was 5'-CGC GGA TCC ATG CCC AGT TCG ACA AA-3' and the reverse primer was 5'-CCG GAA TTC CTC TGG TGG CGC TTC A-3'. After PCR, the BTEB2 cDNA was subcloned 
into the pDC315 vector (Microbix Systems Inc., Toronto, ON, Canada) between the EcoRI and BamHI cloning sites to construct pDC315-As-BTEB2. The pDC315-As-BTEB2 construction was confirmed by sequencing and the recombinant plasmid was co-transfected into 293 cells along with a pBHGlox $\Delta \mathrm{E} 1,3 \mathrm{Cre}$ plasmid containing the $\Delta \mathrm{E} 1$ adenoviral genome. The viruses (Ad-As-BTEB2) were amplified in 293 cells, purified by gradient centrifugation and titrated as previously described (10). An adenovirus coding for $\beta$-galactosidase (Ad-LacZ) was used as a control.

Rat artery balloon injury model. Adult male Wistar rats (obtained from the local animal center) weighing 300-400 g were anesthetized with pentobarbital and heparinized with heparin sodium. Balloon denudation was performed six times in the left common carotid artery with a 2-French catheter (Cordis, Miami, FL, USA) as previously described (11). Rats were randomly divided into four groups $(n=6)$. Rats in the uninjured group $(n=6)$ were not injured with the balloon. Rats in the injured ( $\mathrm{n}=6)$, Ad-LacZ $(\mathrm{n}=6)$ and Ad-As-BTEB2 $(n=6)$ groups received no treatment, a suspension of Ad-LacZ $\left(1 \times 10^{8} \mathrm{pfu}\right)$ or a suspension of Ad-As-BTEB2 $\left(1 \times 10^{8} \mathrm{pfu}\right)$, respectively. The suspension was loaded within a 24-G indwelling needle, which was injected into the injured arterial segment and allowed to incubate for $30 \mathrm{~min}$. Rats were euthanized 21 days after injury. All experimental procedures were performed in accordance with protocols approved by the Institutional Animal Care and Research Advisory Committee.

Vascular SMC culture. Vascular SMCs were obtained from the thoracic aorta of mice and cultured using a tissue explant method as previously described (12). Vascular SMCs were grown in high-glucose Dulbecco's modified Eagle's medium (DMEM; Hyclone, Logan, UT, USA) supplemented with $10 \%$ fetal calf serum (FBS; Hyclone). Cultures were maintained at $37^{\circ} \mathrm{C}$ in a $5 \% \mathrm{CO}_{2}$ humidified incubator. The smooth muscle phenotype of cultured cells was verified by positive immunofluorescence for SM $\alpha$ A. Vascular SMCs cultured in 6-well plates were divided into the following groups: control, Ad-LacZ $\left(1 \times 10^{6} \mathrm{pfu} /\right.$ well $)$ and Ad-As-BTEB2 $\left(1 \times 10^{6} \mathrm{pfu} / \mathrm{well}\right)$. The cells were harvested $72 \mathrm{~h}$ after transfection.

Evaluation of reendothelialization. Reendothelialization was assessed by staining the de-endothelialized areas of the carotid artery with Evans blue dye as previously described (13). In brief, $1 \mathrm{ml}$ of $0.5 \%$ dye solution was injected into the tail vein of the rats under general anesthesia. After the rat was perfused with fixation solution for $30 \mathrm{~min}$, the injured vascular segment was dissected and viewed under a microscope. The percentage of the blue-stained denuded area to the total endothelial area was calculated using a computer-assisted morphometric analysis system (Image Pro Plus 4.5 software; Media Cybernetics, Carlsbad, CA, USA).

Histopathological analysis. Carotid arteries were harvested, embedded, sectioned and mounted on glass slides. Slides were stained with H\&E and analyzed using a computer-assisted morphometric analysis system (Image Pro Plus 4.5 software; Media Cybernetics). The intima/media ratio was calculated.
Immunohistochemical analysis. Paraffin-embedded arteries were cut into cross-sections $(5 \mu \mathrm{m})$, dewaxed and rehydrated. Vascular SMCs were cultured on glass coverslips. Vessel sections and vascular SMCs were placed in a humidified chamber and incubated with the following antibodies overnight: rabbit anti-BTEB2, anti-PCNA, anti-AT1, antiPDGF-BB, anti-SM $\alpha$ A and anti-SMemb (1:200 dilution; Santa Cruz Biotechnology, Santa Cruz, CA, USA). Sections were washed and incubated with a goat biotinylated antirabbit secondary antibody (1:200 dilution; Boster Co., Ltd., Wuhan, China), followed by incubation with streptavidinbiotin-peroxidase reagents from the SABC kit (Boster Co., Ltd.). A diaminobenzidine stain kit (Boster Co., Ltd.) was used to detect a positive reaction by producing a brown color. Quantization of the protein expression involved use of an automated image analysis system (Image Pro Plus 4.5 software; Media Cybernetics) and the positively stained cells or area were measured and expressed as the mean percentage of the neointimal area in at least 10 high-power fields (magnification, $\mathrm{x} 400)$.

Cell proliferation assay. Vascular proliferation of SMCs was measured using the methyl thiazolyl tetrazolium (MTT) assay (14). Vascular SMCs from control, Ad-LacZ and Ad-AsBTEB2 groups were incubated with 0.5\% MTT in DMEM at $37^{\circ} \mathrm{C}$ for $4 \mathrm{~h}$ and then dissolved in $150 \mu \mathrm{l}$ DMSO. The absorbance at $570 \mathrm{~nm}$ was read on a multiwall scanning spectrophotometer (Beckman Coulter, Fullerton, CA, USA).

Flow cytometry. Vascular SMCs were harvested at various times with trypsin-ethylenediamine tetraacetic acid (EDTA), washed with PBS and fixed in $70 \%$ ethanol at $4^{\circ} \mathrm{C}$. Nuclear DNA was stained with a reagent containing propidium iodide $(50 \mu \mathrm{g} / \mathrm{ml})$ and DNase-free RNase $(2 \mathrm{U} / \mathrm{ml})$ before being examined with a fluorescence-activated cell sorter. The population of nuclei in each phase of the cell cycle was determined using CellFIT DNA analysis software (Becton Dickenson, San Jose, CA, USA) (15).

Semi-quantitative RT-PCR. Total RNA was extracted from cultured vascular SMCs using Tripure reagent (Roche Diagnostics Corp., Indianapolis, IN, USA) (16). Total RNA $(3 \mu \mathrm{g})$ was reverse-transcribed using an RT system (Promega, Madison, WI, USA). The cDNA was amplified by PCR using Platinum Taq-DNA polymerase (Clontech, Palo Alto, CA, USA) following a standard semi-quantitative RT-PCR technique. The primers used for the amplification were as follows: SM $\alpha$ A sense 5'-CCT GAA GTA TCC GAT AGA AC-3', SM $\alpha$ A antisense 5'-GCC GAC TCC ATT CCA ATG AA-3' (product 618 bp); SMemb sense 5'-TCA AGT CCA AGT TCA AGG CCA-3', SMemb antisense 5'-TGT CGT CAT CTG ACA GCT CCA-3' (product 456 bp); GAPDH-1 sense 5'-ATC ACC ATC TTC CAG GAG CG-3', GAPDH-1 antisense 5'-TAG GAA CAC GGA AGG CCA TG-3' (product 490 bp); and GAPDH-2 sense 5'-CAT GGT CTA CAT GTT CCA GTC-3', GAPDH-2 antisense 5'-GGC TAA GCA GTT GGT GGT GC-3' (product $349 \mathrm{bp}$ ). The PCR products were visualized after electrophoresis in a 1.5\% agarose gel (Bio-Rad, CA, USA) with ethidium bromide $(0.5 \mu \mathrm{g} / \mathrm{ml}$; Sigma, St. Louis, MO, USA). 
A

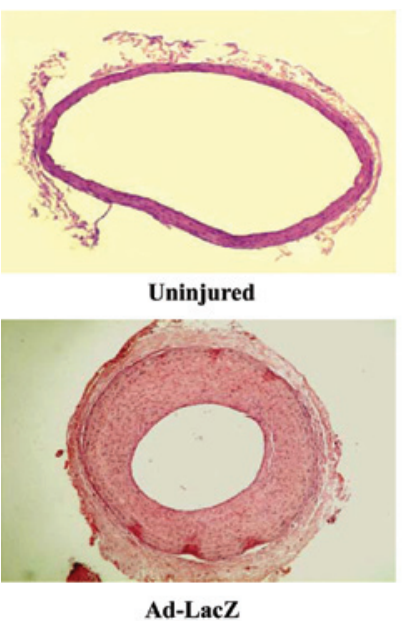

B

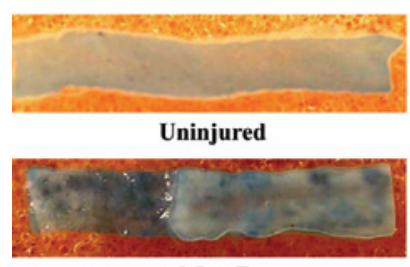

Ad-LacZ

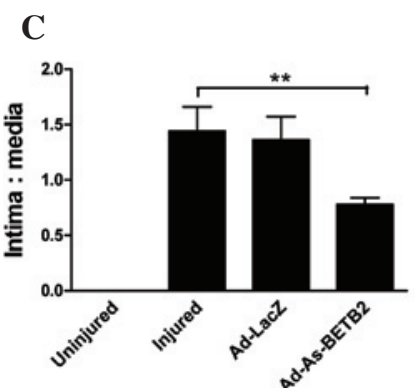

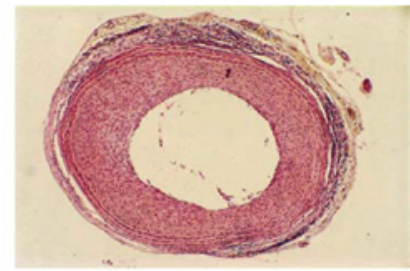

Injured

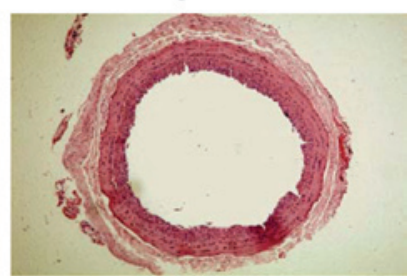

Ad-As-BTEB2

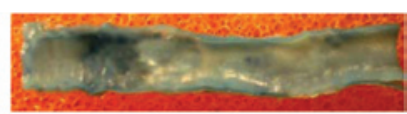

Injured

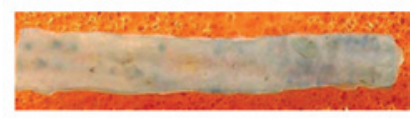

Ad-As-BTEB2

D

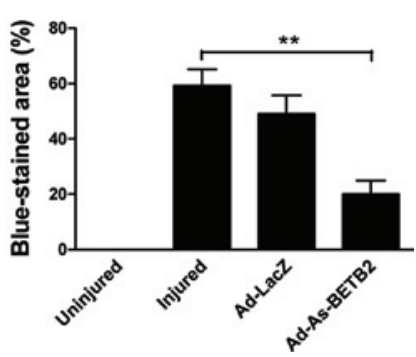

Figure 1. Knockdown of BTEB2 attenuates intimal hyperplasia of carotid arteries 21 days after injury. (A) Representative H\&E-stained cross-sections of carotid arteries from rats in uninjured, injured, Ad-LacZ-treated and Ad-As-BTEB2-treated groups. (B) Photographs showing Evans blue staining of carotid arteries from rats in uninjured, injured, Ad-LacZ-treated and Ad-As-BTEB2-treated groups. (C) The ratios of the intimal area to the medial area of the carotid arteries from the four groups ( $n=6$ for each group) were calculated. Data are expressed as the mean $\pm \mathrm{SD},{ }^{* * *} \mathrm{p}<0.01$. (D) The percentage of blue-stained area within the carotid wall from the four groups ( $n=6$ for each group) were calculated. Data are expressed as the mean $\pm \mathrm{SD},{ }^{* *} \mathrm{p}<0.01$.

Statistical analysis. Data are presented as the mean \pm standard deviation (SD). Comparisons between groups were determined by one-way ANOVA with the Student's post hoc t-test (SPSS Inc., Chicago, IL, USA). $\mathrm{p}<0.05$ indicated significance.

\section{Results}

Knockdown of BTEB2 attenuates intimal hyperplasia. We investigated whether the knockdown of BTEB2 suppressed neointimal hyperplasia in vivo using a rat carotid artery balloon-injured model. At 21 days after balloon-injury, histological analysis revealed that the rats with balloon-injury presented significant levels of neointimal hyperplasia in the carotid artery compared to the uninjured group (Fig. 1A and C). Notably, balloon-injured rats receiving Ad-As-BTEB2

A
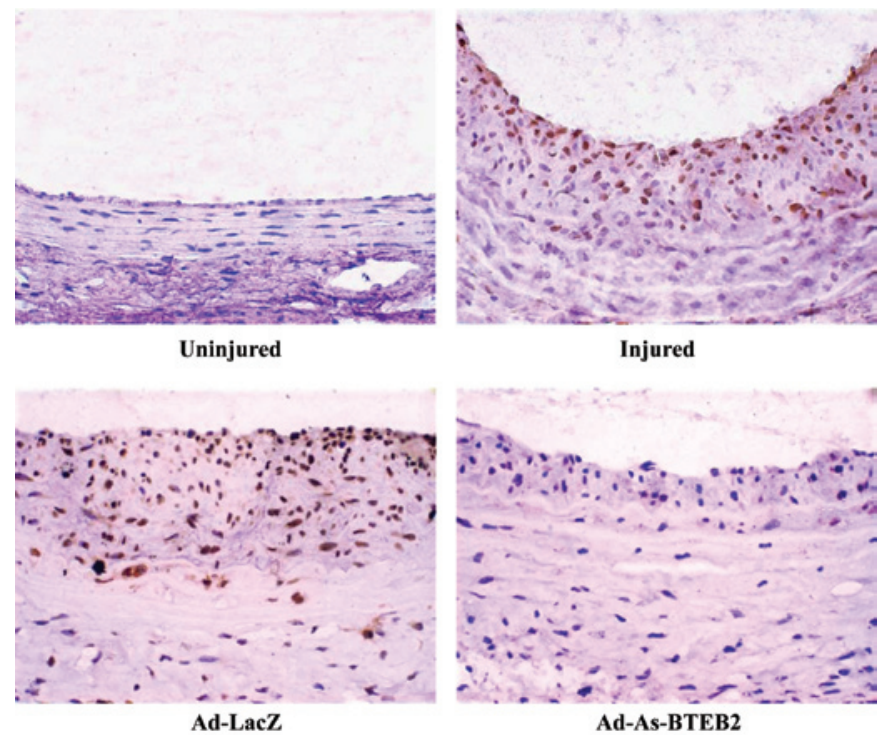

B

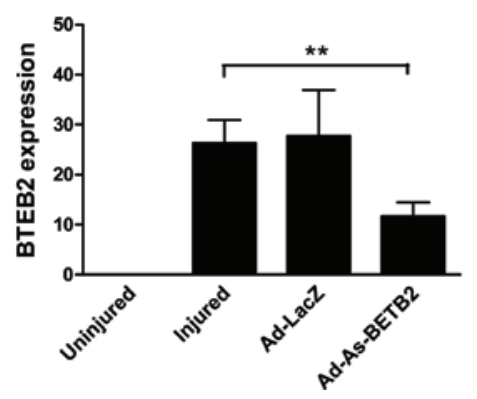

Figure 2. Expression of BTEB2 in carotid arteries. (A) Protein expression of BTEB2 in carotid arteries from rats in uninjured, injured, Ad-LacZ-treated and Ad-As-BTEB2-treated groups were detected by immunohistochemistry (brown represents a positive stain). (B) Quantification for the expression of AT2R in carotid arteries ( $\mathrm{n}=6$ for each group). Data are expressed as the mean $\pm \mathrm{SD}, \stackrel{* *}{\mathrm{p}}<0.01$.

showed a significant reduction of neointimal area (expressed as the ratio of the intimal area to the medial area) compared to non-treated rats (injured group) or rats receiving Ad-LacZ (p<0.01, Fig. 1A and C).

Evans blue staining was used to identify the remaining endothelium-denuded sites. No blue-stained areas were observed in the uninjured artery, but significant blue-stained areas were found in the injured vessel (Fig. 1B and D). Moreover, rats receiving Ad-As-BTEB2 showed a significant reduction in blue-stained areas compared to rats receiving Ad-LacZ (p<0.01, Fig. 1B and D).

Expression of BTEB2 in arteries. Protein expression of BTEB2 was undetectable by immunohistochemistry in carotid arteries from uninjured rats (Fig. 2A and B). Balloon-injury significantly stimulated the expression of BTEB2 (Fig. 2A and B). Immunohistochemistry showed a markedly lower level of BTEB2 protein expression in the Ad-As-BTEB2 group relative to the Ad-LacZ group ( $<<0.01$, Fig. 2A and B). Experiments in vitro demonstrated similar results (data not shown).

Knockdown of BTEB2 suppresses vascular SMC proliferation. We evaluated vascular SMC proliferation using the 

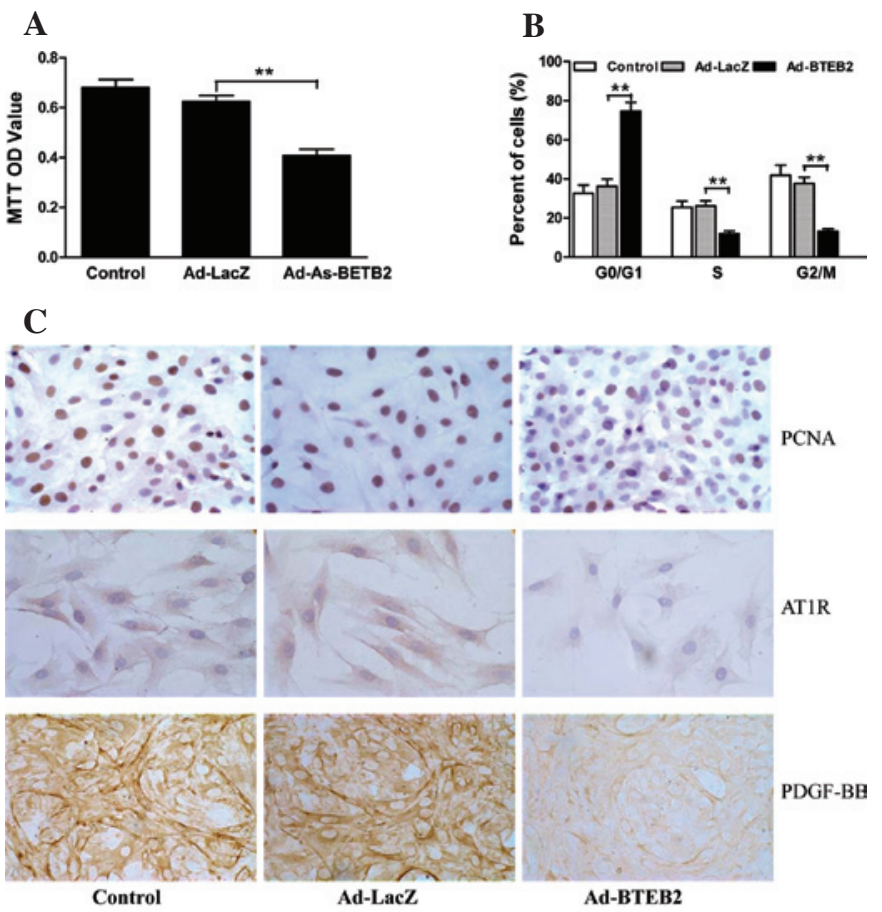

D
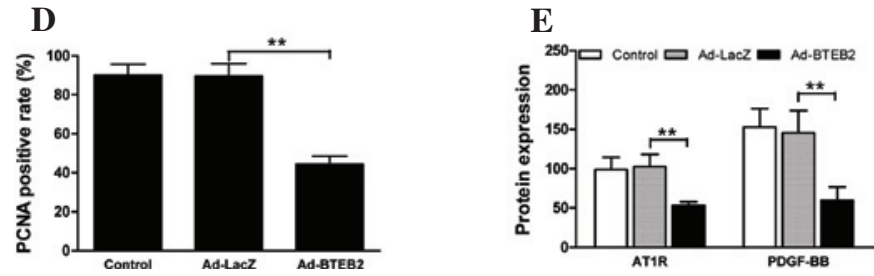

Figure 3. Knockdown of BTEB2 suppresses vascular SMC proliferation Quiescent vascular SMCs were treated with vehicle (control group), Ad-LacZ or Ad-As-BTEB2, and stimulated with $10 \%$ FBS for $72 \mathrm{~h}$. (A) Cell numbers were determined by the MTT OD value. Data are expressed as the mean \pm SD, ${ }^{* *} \mathrm{p}<0.01$. (B) The percentages of cells in the G0/G1, S and G2/M phases of the cell cycle were determined using CellFIT DNA analysis software. Six samples were analyzed in each group and values represent the mean $\pm \mathrm{SD},{ }^{* *} \mathrm{p}<0.01$. (C) Representative immunohistochemistry for PCNA, AT1R and PDGF-BB (brown stain) in cultured vascular SMCs treated with vehicle (control group), Ad-LacZ or Ad-As-BTEB2. (D) The PCNA-positive rate in cultured vascular SMCs was calculated. Values represent the mean $\pm S D,{ }^{* *} \mathrm{p}<0.01$ ( $\mathrm{n}=6$ for each group). (E) Quantification for the expression of AT1R and PDGF-BB in cultured vascular SMCs. Values represent the mean $\pm \mathrm{SD},{ }^{* *} \mathrm{p}<0.01$ ( $\mathrm{n}=6$ for each group).

MTT assay. Transfection with Ad-As-BTEB2 significantly suppressed SMC proliferation compared to Ad-LacZ-treated cells $(\mathrm{p}<0.01$, Fig. 3A). Treatment with Ad-As-BTEB2 increased the cell population at the G0/G1 phase by $42.5 \%$, decreased the cell population at the S phase by $14.3 \%$, and decreased the cell population at the G2/M phase by $24.4 \%$ when compared to the Ad-LacZ-treated group $(\mathrm{p}<0.01$, Fig. $3 \mathrm{~B}$ ), indicating that knockdown of BTEB2 induced cell cycle arrest at the G0/G1 phase. The ratio of PCNA-positive cells in the intima was significantly less in Ad-As-BTEB2-treated vascular SMCs than in Ad-LacZ-treated cells ( $<<0.01$, Fig. 3C and D). In addition, Ad-As-BTEB2 transfection significantly attenuated the protein expression of AT1R and PDGF-BB in cultured vascular SMCs (both p<0.01, Fig. $3 \mathrm{C}$ and E).

Knockdown of BTEB2 modulates vascular SMC phenotype. We observed changes in the protein and mRNA levels of
A
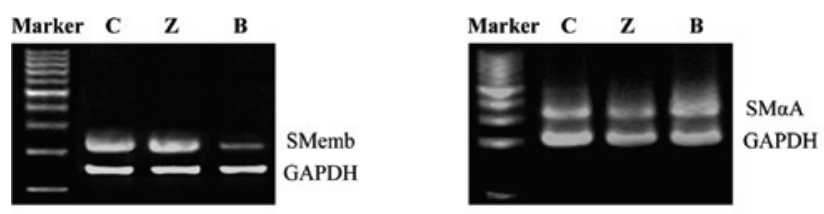

\section{B}
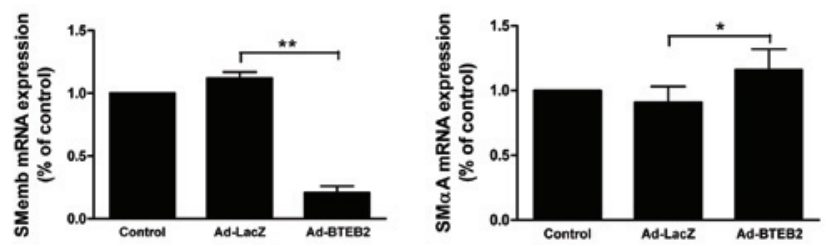

C
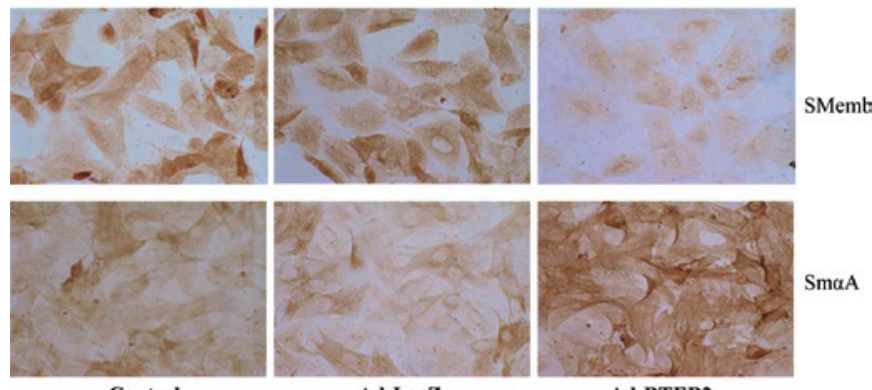

D
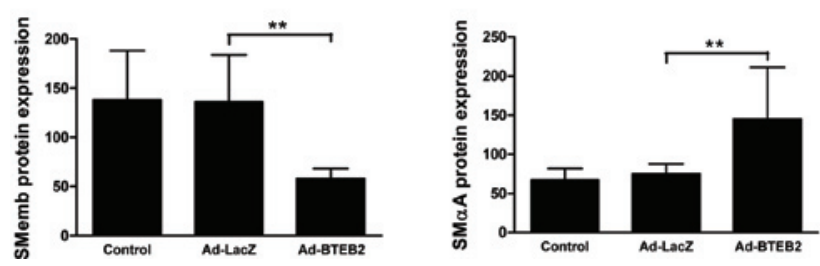

Figure 4. Knockdown of BTEB2 modulates vascular SMC phenotype. (A) Representative RT-PCR for the expression of SMemb and SM $\alpha$ A in cultured vascular SMCs. C, control; Z, Ad-LacZ; B, Ad-As-BTEB2. (B) The target mRNA/GAPDH ratio is shown in the bar graph. ${ }^{* *} \mathrm{p}<0.01(\mathrm{n}=6$ for each group). (C) Protein expression of SMemb and $\mathrm{SM} \alpha \mathrm{A}$ in cultured vascular SMCs was detected by immunohistochemistry (brown represents a positive stain). (D) Quantification for the expression of SMemb and SM $\alpha \mathrm{A}$ in cultured vascular SMCs. ${ }^{* *} \mathrm{p}<0.01$ ( $\mathrm{n}=6$ for each group).

SMemb and SM $\alpha$ A in cultured vascular SMCs. The mRNA expression of SMemb, a marker of undifferentiated proliferating vascular SMCs, was significantly down-regulated compared to the Ad-LacZ treatment. By contrast, SM $\alpha \mathrm{A}$, a marker for differentiated contractile SMCs, was markedly up-regulated by Ad-As-BTEB2 transfection compared to the Ad-LacZ treatment (both p $<0.01$, Fig. $4 \mathrm{~A}$ and B). Immunohistochemistry for SMemb and $\mathrm{SM} \alpha \mathrm{A}$ protein levels showed similar results (both $\mathrm{p}<0.01$, Fig. $4 \mathrm{C}$ and D).

\section{Discussion}

The present results identify a critical role for BTEB2 in neointimal formation in a rat artery balloon injury model, suggesting a potential role for BTEB2 knockdown in the suppression of neointimal development. Meanwhile, we have demonstrated that the knockdown of BTEB2 inhibits vascular 
SMC proliferation concurrent with the down-regulation of PCNA, AT1R and PDGF-BB. We also demonstrated that BTEB2 knockdown stimulates phenotypic changes in vascular SMCs, increasing dedifferentiation markers and decreasing differentiation markers. The inhibitory role of BTEB2 in neointimal formation may be attributed to the suppression of vascular SMC proliferation and subsequent phenotypic cellular changes.

Previous experiments have demonstrated that BTEB2 is down-regulated during aortic development and is re-induced after balloon injury (6), suggesting that BTEB2 may be involved in the development of neointimal formation. The present study demonstrated that the knockdown of BTEB2 significantly reduced balloon injury-induced neointimal formation, indicating that BTEB2 may serve as powerful stimulus of neointimal hyperplasia. Thus, the down-regulation of BTEB2 may become a novel strategy for the prevention of vascular restenosis after PCI.

Neointimal hyperplasia is attributed to the proliferation and phenotypic modulation of vascular SMCs. Previous studies have suggested that vascular SMC proliferation was associated with the up-regulation of BTEB2 (7). The present findings further confirm the potential role of BTEB2 in vascular SMC proliferation. Phenotypic modulation of vascular SMCs is associated with pathological vascular processes. Whereas the contractile phenotype is characteristic of quiescent differentiated SMCs, the synthetic (or activated) phenotype reflects a dedifferentiated and proliferative SMC phenotype. Previous results showed that BTEB2 may be a possible molecular marker of the phenotypic modulation of vascular SMCs. The present study suggests that BTEB2 knockdown promotes the proliferation of vascular SMCs and a dedifferentiated phenotype.

In summary, our data show that the knockdown of BTEB2 suppresses neointimal hyperplasia in a rat artery balloon injury model. BTEB2 appears to be a potential target for the prevention of restenosis after vascular interventions.

\section{Acknowledgements}

This study was supported by the Foundation for the Talents by the General Hospital of PLA Chengdu Military Area Command (to B. Tang), and by grants from the Medical Scientific Research Program of PLA Chengdu Military Area Command during the 11th Five-Year Plan Period (to D. Yang, no. MB09023) and the Science and Technology Project of Sichuan Province (to D. Yang, no. 2010JY0028).

\section{References}

1. Lin GA, Dudley RA and Redberg RF: Cardiologists' use of percutaneous coronary interventions for stable coronary artery disease. Arch Intern Med 167: 1604-1609, 2007.

2. Corbett SJ, Cosgrave J, Melzi G, et al: Patterns of restenosis after drug-eluting stent implantation: insights from a contemporary and comparative analysis of sirolimus- and paclitaxel-eluting stents. Eur Heart J 27: 2330-2337, 2006.

3. Li D, Ma S, Li G, et al: Endovascular stent implantation for isolated pulmonary arterial stenosis caused by Takayasu's arteritis. Clin Res Cardiol 99: 573-575, 2010.

4. Casscells W: Migration of smooth muscle and endothelial cells. Critical events in restenosis. Circulation 86: 723-729, 1992.

5. Lafont A, Guzman LA, Whitlow PL, Goormastic M, Cornhill JF and Chisolm GM: Restenosis after experimental angioplasty. Intimal, medial, and adventitial changes associated with constrictive remodeling. Circ Res 76: 996-1002, 1995.

6. Watanabe N, Kurabayashi M, Shimomura Y, et al: BTEB2, a Kruppel-like transcription factor, regulates expression of the SMemb/nonmuscle myosin heavy chain B (SMemb/NMHC-B) gene. Circ Res 85: 182-191, 1999.

7. Ogata T, Kurabayashi M, Hoshino Y, et al: Inducible expression of basic transcription element-binding protein 2 in proliferating smooth muscle cells at the vascular anastomotic stricture. J Thorac Cardiovasc Surg 119: 983-989, 2000.

8. Hoshino Y, Kurabayashi M, Kanda T, et al: Regulated expression of the BTEB2 transcription factor in vascular smooth muscle cells: analysis of developmental and pathological expression profiles shows implications as a predictive factor for restenosis. Circulation 102: 2528-2534, 2000.

9. Kawai-Kowase K, Kurabayashi M, Hoshino Y, Ohyama Y and Nagai R: Transcriptional activation of the zinc finger transcription factor BTEB2 gene by Egr-1 through mitogen-activated protein kinase pathways in vascular smooth muscle cells. Circ Res 85: 787-795, 1999.

10. Lakka SS, Rajan M, Gondi C, et al: Adenovirus-mediated expression of antisense MMP-9 in glioma cells inhibits tumor growth and invasion. Oncogene 21: 8011-8019, 2002.

11. Matsumae H, Yoshida Y, Ono K, et al: CCN1 knockdown suppresses neointimal hyperplasia in a rat artery balloon injury model. Arterioscler Thromb Vasc Biol 28: 1077-1083, 2008.

12. Cheng XW, Kuzuya M, Sasaki T, et al: Green tea catechins inhibit neointimal hyperplasia in a rat carotid arterial injury model by TIMP-2 overexpression. Cardiovasc Res 62: 594-602, 2004.

13. Kikuchi S, Umemura K, Kondo K, Saniabadi AR and Nakashima M: Photochemically induced endothelial injury in the mouse as a screening model for inhibitors of vascular intimal thickening. Arterioscler Thromb Vasc Biol 18: 1069-1078, 1998.

14. Cheng B, Song J, Zou Y, et al: Responses of vascular smooth muscle cells to estrogen are dependent on balance between ERK and p38 MAPK pathway activities. Int J Cardiol 134: 356-365, 2009.

15. Lien HM, Lin HW, Wang YJ, et al: Inhibition of anchorageindependent proliferation and G0/G1 cell-cycle regulation in human colorectal carcinoma cells by 4,7-dimethoxy-5-methyl1,3-benzodioxole isolated from the fruiting body of Antrodia camphorate. Evid Based Complement Alternat Med: March, 2009 (E-pub ahead of print).

16. Yang D, Ma S, Tan Y, et al: Increased expression of calpain and elevated activity of calcineurin in the myocardium of patients with congestive heart failure. Int J Mol Med 26: 159-164, 2010. 